\title{
Distribuição, evolução e produção científica dos grupos de pesquisa em atividade física e saúde do Brasil
}

\section{Distribution, evolution and scientific research groups on physical activity and health in Brazil}

\author{
Amanda Santos ${ }^{1,4}$ \\ Letícia Lemos Ayres da Gama Bastos ${ }^{1,3}$ \\ Andrezza Aparecida Aleixo ${ }^{1,4}$ \\ Thaís Reis Silva de Paulo ${ }^{1,2}$ \\ Edmar Lacerda Mendes ${ }^{1}$ \\ 1. Programa de Mestrado em Educação Física \\ da Universidade Federal do Triângulo Mineiro. \\ Uberada, MG, Brasil. \\ 2. CAPES-DS \\ 3. FAPEMIG. Belo Horizonte, MG, Brasil. \\ 4. REUNI
}

\begin{abstract}
Resumo
O presente estudo objetivou verificar a distribuição geográfica, evolução e produção científica dos Grupos de Pesquisa em Atividade Física e Saúde (GPAFS) no Brasil. Realizou-se busca sistemática na base corrente e nos censos do Diretório de Grupos de Pesquisa do Conselho Nacional de Desenvolvimento Científico e Tecnológico (CNPq), utilizando a frase exata "Atividade Física e Saúde" e Ciências da Saúde como filtro. Foram encontrados 141 GPAFS. A produção científica dos grupos foi obtida pela análise do Currículo Lattes de seus líderes. Analisaram-se os Censos a partir do ano 2000, segundo o ano de formação dos GPAFS. Divididos geograficamente, $24,82 \%$ dos GPAFS situam-se na região Sudeste, 38,30\% Sul, 23,40\% Nordeste, 5,67\% Norte e 7,80\% Centro-Oeste. Segundo a localização e a produção dos líderes, temos respectivamente, 23,02\% e 32,70\% no Sudeste, $34,67 \%$ e $37,10 \%$ Sul, $25,28 \%$ e $21,32 \%$ Nordeste, $6,95 \%$ e 2,36\% Norte e 10,08\% e 6,52\% Centro-Oeste. Quanto ao período de formação, 9,93\% dos GPAFS surgiram entre 2000 e $2002,8,51 \%$ entre 2002 e 2004, 15,60\% entre 2004 e 2006, 17,73\% entre 2006 e 2008 e $19,86 \%$ entre 2008 e 2010. Das seis grandes áreas da saúde que estudam o tema, $72,34 \%$ dos GPAFS pertencem a Educação Física. Os dados apresentam discrepância do número de GPAFS, principalmente em relação às regiões Centro-Oeste e Norte, além da concentração da produção científica no eixo Sul-Sudeste. Políticas de incentivo à formação de jovens mestres e doutores devem ser estimuladas de forma igualitária no Brasil a fim de suprir as desigualdades entre as regiões.
\end{abstract}

Palavras-chave: Grupos de Pesquisa; Atividade física; Saúde.
ENDEREÇOPARA CORRESPONDÊNCIA

\section{Amanda Santos}

Rua Tenente Wenceslau de Oliveira, 159

CEP 38025-400 - Uberaba, MG

e-mail:amanda637@hotmail.com

$\begin{array}{ll}\text { - Recebido: } & 27 / 03 / 2012 \\ \text { - Re-submissão: } & 22 / 06 / 2012 \\ \text {-Aceito: } & 13 / 07 / 2012\end{array}$

\begin{abstract}
This study aimed to assess the geographic distribution, evolution and Scientific Research Groups on Physical Activity and Health (GPAFS) in Brazil. We conducted a systematic search based on the current census of Directory of Research Groups of the National Council of Technological and Scientific Development (CNPq), using the exact phrase "Physical Activity and Health" and Health Sciences as a filter. We found 141 GPAFS. The scientific group was obtained by analyzing the Curriculum Lattes their leaders. We analyzed the Census from the year 2000, following the onset year of GPAFS. When divided geographically, $24.82 \%$ of GPAFS are located in Southeast, $38.30 \%$ in South, $23.40 \%$ in North-East, $5.67 \%$ in North and $7.80 \%$ in Midwest Region. As for the groups and the productions of their leaders have respectively $23.02 \%$ and $32.70 \%$ in the Southeast, $34.67 \%$ and $37.10 \%$ South, $25.28 \%$ and $21.32 \%$ North-East, $6.95 \%$ and $2.36 \%$ North and $10.08 \%$ and $6.52 \%$ Midwest. As regards the rearing period, $9.93 \%$ of GPAFS emerged between 2000 and 2002, 8.51\% between 2002 and 2004, 15.60\% between 2004 and 2006, 17.73\% between 2006 and 2008, and 19.86\% between 2008 and 2010 . Of the six major areas of health who study the issue, $72.34 \%$ of GPAFS owned Physical Education. The data show a discrepancy in the number of GPAFS, especially in relation to the Midwest and North region, and the concentration of scientific production in SouthSoutheast region. Policies to promote training of young teachers and doctors should be encouraged equally in Brazil to address the inequalities between regions. Keywords: Activity, Research; Physical Activity; Health.
\end{abstract}




\section{INTRODUÇÃO}

O processo da globalização trouxe consigo inevitáveis transformações no cotidiano das pessoas, sobretudo após as marcantes transições demográficas, epidemiológicas, nutricionais e tecnológicas vivenciadas nos últimos cinqüenta $\operatorname{anos}^{1}$. Como consequência da transição da sociedade tradicional para a moderna, as principais doenças não-transmissíveis (doenças cardiovasculares, câncer, doença pulmonar obstrutiva crônica e diabetes) estão relacionadas a fatores de risco evitáveis como tabagismo, dieta não saudável e comportamento sedentário ${ }^{2}$. Assim, o constante processo de informatização da "era mecanicista" ${ }^{3}$, tem proporcionado importantes mudanças nos hábitos de vida da humanidade, onde a rápida ascensão das doenças não-transmissíveis representam desafios para a saúde pública em todo o mundo.

Entretanto, se por um lado, a tecnologia facilita a realização das tarefas diárias, ao custo de comportamentos hipocinéticos, por outro, propicia a rápida veiculação e acesso a conhecimentos nas áreas científicas, em especial da Educação Física e Esportes, o avanço da medicina, indústria, entre outros $^{4}$.

Como conseqüência das constantes mudanças supracitadas, esforços têm sido destinados à produção do conhecimento com a finalidade de compreender o atual perfil da população. Neste contexto, investigações que inserem atividade física como estratégia, tanto de prevenção e promoção como de reabilitação da saúde ganham importância, com destaque aos trabalhos que evidenciam associação entre o elevado nível de atividade física ou aptidão física ${ }^{5}$ e redução no risco de doenças crônico não-transmissíveis ${ }^{6}{ }^{7}$.

Nesse sentido, esta linha de pesquisa vem se apoiando cientificamente na grande área saúde, principalmente no campo da Educação Física, pois desde que esta começou a se preocupar com seu objeto de estudo começa alavancar sua produção acadêmica ${ }^{8}$, recebendo assim, ampla atenção das agências de fomento à pesquisa e dos cursos de graduação e pós-graduação das universidades do país ${ }^{9}$.

Em 2010, estavam registrados na área de Educação Física 502 cursos de graduação, dos quais 240 na região Sudeste, 95 no Sul, 84 no Nordeste, 51 no Centro-Oeste e 32 no Norte ${ }^{9}$. No mesmo período, 23 programas de pós-graduação stricto sensu encontravam-se cadastrados na Coordenação de Aperfeiçoamento de Pessoal de Nível Superior (CAPES), sendo 13 na região Sudeste, seis no Sul, dois no Centro-Oeste e dois no Nordeste?.

Portanto, o presente estudo teve os objetivos de verificar e caracterizar a distribuição geográfica, evolução e produção científica dos Grupos de Pesquisa em Atividade Física e Saúde (GPAFS) no Brasil.

\section{MÉTODOS}

Para a coleta de informações para identificação e caracterização da produção científica dos GPAFS que desenvolvem pesquisa na interface "atividade física e saúde", realizou-se busca sistemática na base corrente do Diretório de Grupos de Pesquisa do Conselho Nacional de Desenvolvimento Científico e Tecnológico (CNPq). Os grupos recuperados foram aqueles que estão na situação de "grupos certificados" pelos dirigentes internacionais de pesquisa.

A busca foi realizada em maio de 2011 por dois pesquisadores. Um deles coletou informações da região Norte, Nordeste e Sudeste e o outro das regiões Sul e Centro-Oeste. Para a busca, foi utilizada a frase exata "atividade física e saúde", filtro para a grande área do grupo: Ciência da Saúde, restringindo os resultados ao tema de interesse, dentro da área de atuação da Educação Física. A busca dos grupos acadêmicos foi realizada sobre o nome do grupo, UF, instituição, área, ano de formação, número de pesquisadores, estudantes, técnicos e linha de pesquisa. A produção dos líderes dos GPAFS do Brasil foi quantificada seguindo três critérios adotados: artigos em periódicos, trabalhos completos publicados em anais de congresso e artigos aceitos para publicação, consultados na plataforma Lattes. Verificou-se também a evolução dos grupos de pesquisa na área de conhecimento Educação Física (GPEF), por ano de formação entre 2000 a 2010, datas disponibilizadas pelo site do CNPq.

Os dados coletados foram armazenados no programa Excel, versão 2007. Para análise dos dados foi empregada estatística descritiva, apresentados em valores absolutos e percentuais.

\section{RESULTADOS}

A Figura 1 apresenta a distribuição dos GPAFS no Brasil. A região Sul apresenta a maior concentração $(38,3 \% ; n=54)$ e distribuição dos GPAFS em funcionamento no país. Em seguida, encontra-se a região Sudeste $(24,8 \% ; n=35)$. Os estados de SP e PR possuem o maior número de grupos. Em seguida, encontra-se a região Nordeste $(23,4 \% ; n=33)$, a região Centro-Oeste $(7,8 \% ; n=11)$ e, por fim, a região Norte $(5,7 \% ; n=8)$. Cinco Estados apresentam apenas um grupo cadastrado (ES, $\mathrm{RO}, \mathrm{TO}, \mathrm{MS}, \mathrm{GO}$ ). Nos estados de Roraima e Amapá, na região Norte, e Maranhão e Piauí, na região Nordeste, não foram encontrados GPAFS cadastrados no CNPq.

A Tabela 1 relaciona os integrantes dos GPAFS: pesquisadores, estudantes e técnicos, envolvidos em cada região do Brasil. A região Sul concentra o maior número de integrantes ( $n=1056)$ em GPAFS no Brasil, seguidos pelas regiões Nordeste $(n=751)$ e Sudeste $(n=699)$. As regiões Centro-Oeste e Norte possuem os menores números relativos e absolutos em relação ao número de integrantes dos GPAFS em todo território brasileiro.

A titulação dos líderes dos GPAFS foi classificada em professor auxiliar, mestre, doutor e doutor com bolsa produtividade do CNPq. Do total de quatro auxiliares envolvidos $25 \%$ $(n=1)$ estão na região Sul, $50 \%(n=2)$ na região Nordeste e $25 \%$ $(n=1)$ na região Norte. Foram encontrados 60 mestres, 15\% $(n=9)$ na região Sudeste, $43,3 \%(n=26)$ na região Sul, 31,7\% $(n=19)$ na região Nordeste, 3,3\% $(n=2)$ na região Norte e 6,7 \% $(n=4)$ na região Centro-Oeste. Doutores são a maioria $(n=137)$, $29,2 \%(n=40)$ na região Sudeste, $33,6 \%(n=46)$ na região Sul, $31,7 \%(n=26)$ na região Nordeste, $8,0 \%(n=11)$ na região Norte e $10,2 \%(n=14)$ na região Centro-Oeste. A estes doutores, soma-se $30,8 \%(n=8)$ no Sudeste, $38,5 \%(n=10)$ no Sul, $23,1 \%$ $(n=6)$ no Nordeste e $7,7 \%(n=2)$ no Centro-Oeste do total de 26 líderes que possuem bolsa produtividade do CNPq.

Acerca da quantificação da produção científica dos líderes dos GPAFS, a qual foi calculada pelo somatório dos artigos publicados em periódicos indexados, trabalhos completos publicados em anais de congressos e artigos aceitos para publicação constantes no currículo dos líderes de GPAFS no Brasil disponíveis na plataforma Lattes, a região Sul possui a maior concentração de produção Sul $(n=2993 / 37,1 \%)$, seguida das regiões Sudeste $(n=2638 / 32,7 \%)$ e Nordeste $(n=1720$ / $21,3 \%)$. As regiões Centro-Oeste ( $n=526$ / 6,5\%) e Norte $(n=190 / 2,4 \%)$ apresentaram os menores valores absolutos e percentuais. 


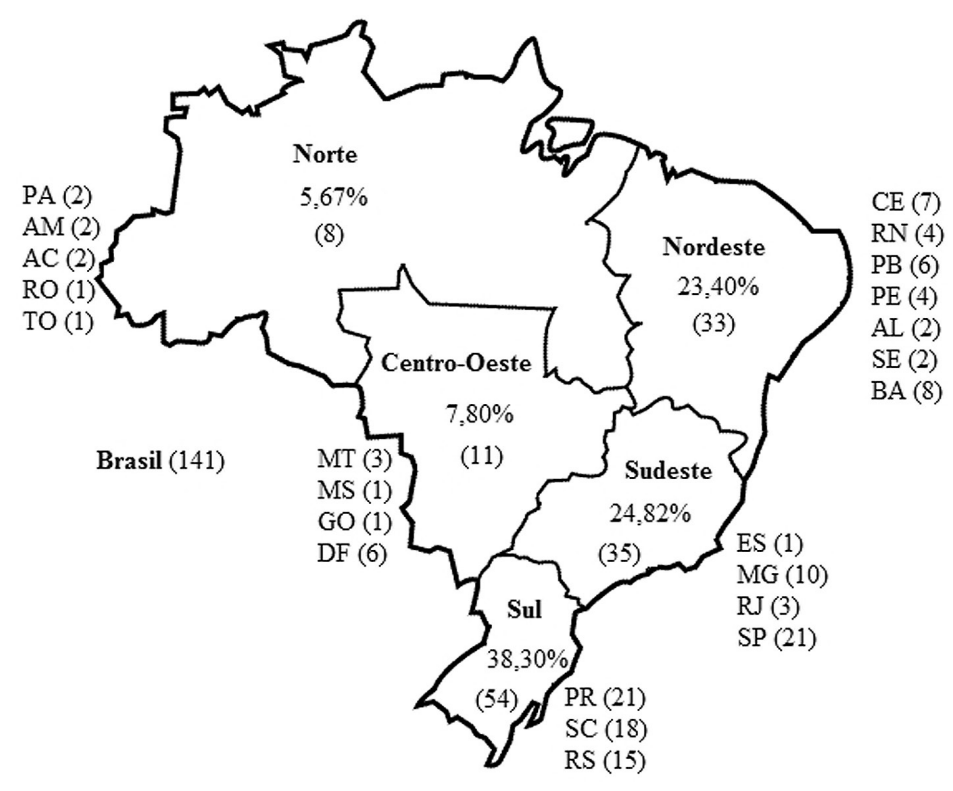

Figura 1

Distribuição geográfica dos Grupos de Pesquisa em Atividade Física e Saúde no Brasil, 2011. Valores percentuais por regiões e frequência nos estados entre parênteses.

A Figura 2 apresenta a produção científica dos líderes dos GPAFS subdivididas entre os três critérios investigados. Os artigos publicados em periódicos são a maioria em todas as regiões $(75,2 \% \pm 7,6 \%)$, seguidos dos trabalhos completos publicados em anais de congresso $(20,7 \% \pm 7,5 \%)$ e artigos aceitos para publicação $(4,1 \% \pm 1,4 \%)$.

Números do Censo em relação aos Grupos de Pesquisa Cadastrados (GPC) no diretório desde 2000 até 2010, a cada dois anos foram apresentados na Tabela 2, relacionando-os à Grande Área de conhecimento Educação Física (GPEF), segundo o ano de formação dos grupos. Nota-se uma crescente de aproximadamente 100 grupos de pesquisa na GPEF a cada censo.

Seguindo este modelo de agrupamento bianual, a Tabela 3 traz os 141 GPAFS encontrados também organizados por ano de formação.

Por fim, temos a informação da quantidade de grupos de pesquisas distribuídos nas seis Grandes Áreas da Saúde que pesquisam o tema de atividade física e saúde. A Educação Física detém a maioria dos GPAFS ( $n=102$ / 72,3\%), seguido da Saúde Coletiva ( $n=19$ / 13,5\%), Fisioterapia e Terapia Ocupacional $(n=10 / 7,1 \%)$. Os menores valores absolutos e percentuais são das áreas da Nutrição $(n=4 / 2,8 \%)$, Enfermagem $(n=3 / 2,1 \%)$ e Medicina $(n=3 / 2,1 \%)$.

\section{DISCUSSÃO}

O presente estudo objetivou identificar e caracterizar os GPAFS no Brasil, bem como quantificar a produção científica relacionada à suas ações entre as regiões brasileiras. Os dados apresentados indicam que os GPAFS e suas produções estão concentrados nas regiões Sul, Sudeste e Nordeste em detrimento das regiões Centro-Oeste e Norte.

A crescente discussão sobre a utilização da atividade física como prevenção e controle de doenças associadas ao sedentarismo tem trazido uma consequente produção de conhecimento na área da atividade física e saúde, uma vez que os GPAFS estão se expandindo ${ }^{10}$. Tais grupos respaldam a formação de pesquisadores, atuando na resolução dos problemas locais identificados por meio de suas pesquisas, que auxiliam na elaboração de políticas públicas e ainda trabalham no intuito de subsidiar os cursos de graduação e pós-graduação captando fomentos de pesquisa para a construção de seus estudos ${ }^{9}$.

No Sul e Sudeste do Brasil há um predomínio dos GPAFS, seguidos das regiões Nordeste, Centro-oeste e Norte. A existência do maior número de Instituições de Ensino Superior (IES), de acordo com o e-MEC - Sistema de Regulação do Ensino Superior - e a vocação destas regiões em investigar o Brasil, 2011.

\begin{tabular}{ccccc}
\hline Região & Pesquisadores (\%) & Estudantes (\%) & Técnicos (\%) & Total (\%) \\
\hline Sudeste & $265\left(23,0^{*}\right)$ & $419\left(25,3^{*}\right)$ & $15\left(17,9^{*}\right)$ & $699\left(24,2^{* *}\right)$ \\
Sul & $399\left(34,7^{*}\right)$ & $618\left(37,3^{*}\right)$ & $39\left(46,4^{*}\right)$ & $1056\left(36,5^{* *}\right)$ \\
Nordeste & $291\left(25,3^{*}\right)$ & $445\left(26,9^{*}\right)$ & $15\left(17,9^{*}\right)$ & $751\left(26,0^{* *}\right)$ \\
Norte & $80\left(7,0^{*}\right)$ & $38\left(2,3^{*}\right)$ & $3\left(3,6^{*}\right)$ & $121\left(4,2^{* *}\right)$ \\
Centro-Oeste & $116\left(10,1^{*}\right)$ & $136\left(8,2^{*}\right)$ & $12\left(14,3^{*}\right)$ & $264\left(9,1^{* *}\right)$ \\
\hline Total no Brasil & $\mathbf{1 1 5 1}(\mathbf{3 9 , 8 *})$ & $\mathbf{1 6 5 6}(\mathbf{5 7 , 3 * *})$ & $\mathbf{8 4}(\mathbf{2 , 9 * *})$ & $\mathbf{2 8 9 1 ( 1 0 0 * * )}$ \\
\hline
\end{tabular}

*Valores percentuais entre regiões. **Valores percentuais entre os integrantes dos GPAFS. 


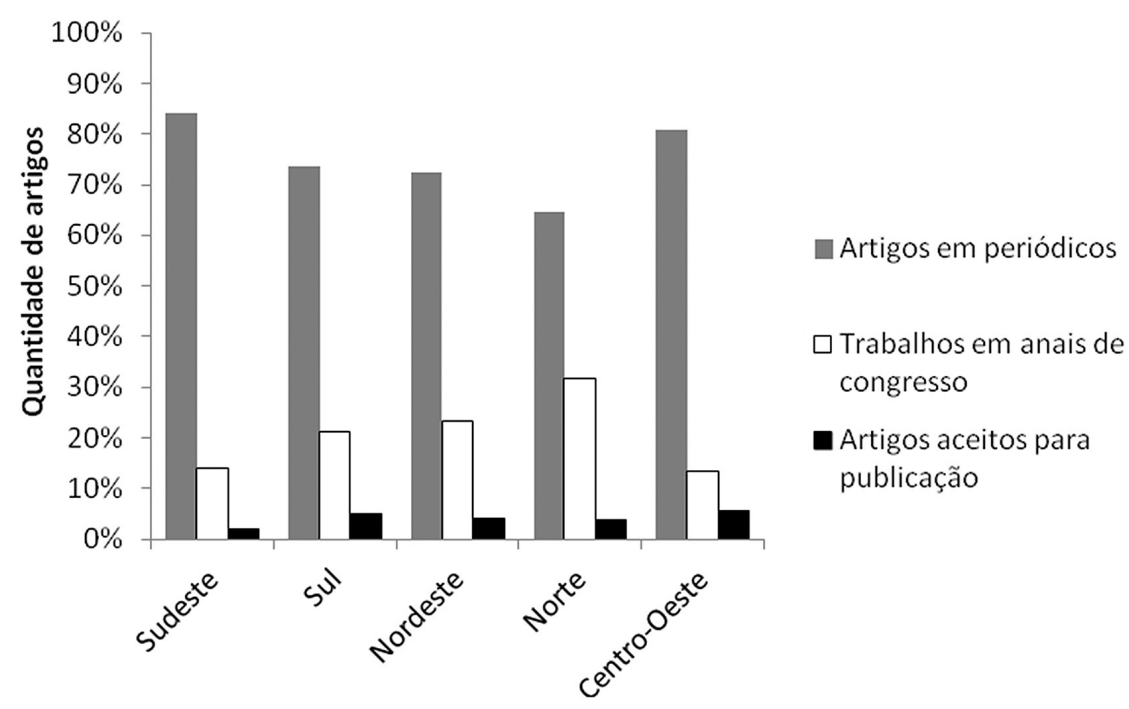

Figura 2

Produção científica dos líderes dos Grupos de Pesquisa em Atividade Física e Saúde no Brasil, 2011.

tema, são fatores decisivos nos números apresentados. Por outro lado, de forma a diminuir discrepâncias entre as regiões no tocante da produção científica, o governo decretou a Lei N. ${ }^{\circ} 10.197$, de 14/02/2001, a qual destina $30 \%$ dos recursos a IES sediadas nas regiões Norte, Nordeste e Centro-Oeste.

No entanto, foi encontrado em nossos resultados que a região Nordeste apresenta, aproximadamente, a mesma proporção de pesquisadores em relação ao Sudeste, porém, não acontece o mesmo para a sua produção. Essa carência de estudos nas outras regiões é preocupante. Pode-se inferir que este fato ocorre devido à maior concentração de doutores na região Sudeste, se comparada à região Nordeste, onde há uma maior oportunidade para a atuação de mestres nas IES.

Dentre os 141 GPAFS encontrados, 72,3\% ( $n=102)$ se concentram no campo da Educação Física. O destaque da Educação Física vem ocorrendo pelo fato desta possibilitar a interação com diferentes áreas, contribuir com o desenvolvimento humano, além de dar suporte na busca da real identidade da área ${ }^{10}$, pois a Educação Física, na tentativa de promover o desenvolvimento integral do ser humano, trabalha

\section{Tabela 2}

Números dos Censos de todos os Grupos de Pesquisas Cadastrados (GPC) no diretório e da Grande Área de conhecimento Educação Física (GPEF), segundo seu ano de formação, de 2000 até 2010.

\begin{tabular}{ccc}
\hline $\begin{array}{c}\text { Ano de } \\
\text { formação }\end{array}$ & $\begin{array}{c}\text { Grupo Pesquisa Cadastrados } \\
\text { (GPC) }\end{array}$ & $\begin{array}{c}\text { Grande Área de Conhecimento EF } \\
\text { (GPEF) }\end{array}$ \\
\hline 2000 & 11760 & $98\left(0,8 \%^{*}\right)$ \\
2002 & 15158 & $196\left(1,3 \%^{*}\right)$ \\
2004 & 19470 & $268\left(1,4 \% \%^{*}\right)$ \\
2006 & 21024 & $304(1,4 \% *)$ \\
2008 & 22797 & $387(1,7 \% *)$ \\
2010 & 27523 & $494(1,8 \% *)$ \\
\hline Total & $\mathbf{1 1 7 7 3 2}$ & $\mathbf{1 7 4 7}$ \\
\hline
\end{tabular}

*Valores percentuais dos GPEF dentro dos GPC no CNPq.

\begin{tabular}{ll}
\hline Ano de Formação & GPAFS \\
\hline Antes de $2000^{*}$ & $17(12,1 \%)$ \\
de 2000 a 2002 & $14(9,9 \%)$ \\
de 2002 a 2004 & $12(8,5 \%)$ \\
de 2004 a 2006 & $22(15,6 \%)$ \\
de 2006 a 2008 & $25(17,7 \%)$ \\
de 2008 a 2010 & $28(19,9 \%)$ \\
Depois de $2010 * *$ & $23(16,3 \%)$ \\
\hline
\end{tabular}

*O grupo mais antigo data 1983

**Até o primeiro semestre de 2011 
interagindo de forma natural e essencial com outras áreas do conhecimento como medicina, fisioterapia, nutrição e saúde coletiva, áreas que possuem objetivos em comum, respeitando os quesitos qualidade de vida, prevenção e promoção de saúde da população.

De acordo com nossos achados, houve crescimento dos GPAFS desde o ano 2000 até a data da coleta, refletido pelo avanço da produção científica em atividade física e saúde no Brasil. Questões como a inclusão da Educação Física na área da saúde juntamente com a inserção de profissionais da área nos cursos de Pós-graduação em Saúde Coletiva e áreas afins contribuíram para as estatísticas apresentadas ${ }^{11}$. Dessa forma, o interesse em investigar atividade física e saúde no meio acadêmico, pode ser reflexo da preocupação global em reforçar a adoção de hábitos saudáveis frente aos agravos à saúde², repetidamente apontados pelos veículos de comunicação científicos e de massa.

A disparidade regional nas publicações permanece corroborando aos achados de Hallal et al. (2007), que utilizaram o descritor "atividade física" com objetivo de investigar a origem das publicações entre as Regiões brasileiras. Os autores verificaram que a maioria dos estudos foram realizados nas regiões Sudeste (43\%) e Sul (36\%) e os demais foram distribuídos entre as regiões Nordeste (11\%), Norte (5\%) e Centro-Oeste (5\%).

Torna-se necessário a busca de políticas de incentivo à formação de mestres e doutores, distribuídos de forma igualitária em todo território nacional a fim de suprir as desigualdades entre as regiões em relação ao financiamento de pesquisas e consequentes produções. Assim, políticas integradas nos pilares de ensino, pesquisa e extensão que sejam capazes de suprir as disparidades regionais e atender a sociedade no âmbito socioeconômico, saúde, educação e esporte devem ser estimuladas.

Ressaltamos que os achados da presente investigação estão restritos a metodologia aplicada. A utilização de dois pesquisadores envolvidos nas coletas de informações por regiões previamente definidas e não de forma independente para posterior cruzamento dos dados aumenta a probabilidade de viés, além da escolha da frase exata e filtros aplicados.

\section{CONCLUSÃO}

O tema atividade física e saúde nunca esteve tão evidenciado como nos últimos anos no Brasil. Os achados apresentados sinalizam discrepância do número de GPAFS, concentrados principalmente nas regiões Sul, Sudeste e Nordeste em relação às regiões Centro-Oeste e Norte, além da concentração da produção científica no eixo Sul-Sudeste.

\section{REFERÊNCIAS}

1. Brito F. Transição demográfica e desigualdades sociais no Brasil. R Bras Est Pop 2008;25:5-26.

2. WHO - World Health Organization. 2008-2013 action plan for the global strategy for the prevention and control of noncommunicable diseases : prevent and control cardiovascular diseases, cancers, chronic respiratory diseases and diabetes. 2008. Geneva: 48.

3. Bento JO. Formação de Mestres e Doutores: Exigências e Competências. Rev Port Cien Desp 2008;8(1):169-83.

4. Pereira RS, Mendes DS, Botti M. Reflexões sobre a visibilidade da produção científica da educação física na era digital. Arquivos em Movimento 2009;5(2):90-108.

5. Matsudo S, Araújo T, Marsudo V, Andrade D, Andrade E, Oliveira LC, et al. Questinário Internacional de Atividade Física (IPAQ): estudo de validade e reprodutibilidade no Brasil. Rev bras ativ fís saúde 2001;6(2):5-18.

6. Lima e Costa MFF, Guerra HL, Firmo JOA, Uchô E. Projeto Bambuí: um Estudo Epidemiológico de Características Sociodemográficas, Suporte Social e Indicadores de Condição de Saúde dos Idosos em Comparação aos Adultos Jovens. Inf Epid SUS 2001;10(4):147-61.

7. Malta DC, Cezário AC, Moura Ld, Morais Neto OLd, Silva Junior JBd. A construção da vigilância e prevenção das doenças crônicas não transmissíveis no contexto do Sistema Único de Saúde. Epid Serv Saúde 2006;15:47-65.

8. Pereira B. As limitações do método científico: implicações para a educação física. Rev paul Educ Fís 1998;12(2):228-48.

9. Mendes EL, Andaki ACR, Andaki Jr. R. Grupos de Pesquisa em atividade Física e Saúde no País: quem são e em que lugares estão localizados. In: VIRTUOSO JS, Editor. Interfaces multidisciplinares na saúde aplicadas na formação do profissional em Educação Física. Uberaba: UFTM, 2011:29-37.

10. Nahas MV, Garcia LMT. Um pouco de história, desenvolvimentos recentes e perspectivas para a pesquisa em atividade física e saúde no Brasil. Rev bras Educ Fís Esp 2010;24:135-48.

11. Hallal PC, Dumith Sde C, Bastos JP, Reichert FF, Siqueira FV, Azevedo MR. [Evolution of the epidemiological research on physical activity in Brazil: a systematic review]. Rev Saúde Pública 2007;41(3):453-60. 